Ivan MACUT

IL COMPITO DELLA STORIA DELLA FILOSOFIA SECONDO FILOSOFO CROATO ALBERTO BAZALA NEL PRIMO VOLUME DELLA SUA OPERA STORIA DELLA FILOSOFIA

The task of the history of philosophy according to Albert Bazala in the first volume of his book History of Philosophy

UDK: 1Bazala,A.

$101.1+13$

$1(091)(497.5)$

Izvorni znanstveni rad

Služba Božja 4I 19.

Original scientific paper

Primlieno: 10/2019.

\title{
Riassunto
}

In questo articolo stiamo esplorando il compito della storia della filosofia secondo il filosofo croato Alberto Bazala, e ciò di cui lui ha scritto nel suo importante lavoro La storia della filosofia. Dopo brevi osservazioni introduttive e nel primo capitolo un breve resoconto della vita e del lavoro di Alberto Bazala, passiamo al secondo capitolo in cui presentiamo schematicamente il lavoro di Bazala intitolato La storia della filosofia. Volume I. La storia della filosofia popolare dei greci. Il terzo, e anche il principale, capitolo affronta il compito della storia della filosofia, come lo vede Bazala, ed è diviso nei seguenti argomenti: L'importanza della storia della filosofia; il doppio compito della storia della filosofia; fattore socio-culturale; tripla interpretazione dei sistemi filosofici; critica del singolo sistema; il valore della storia della filosofia; l'importanza della storia della filosofia; problemi filosofici e, infine, la divisione della storia della filosofia. Nella conclusione del documento, descriviamo sistematicamente ed evidenziamo i punti principali dell'approccio positivo di Bazala alla storia della filosofia, e delineammo brevemente la sua relazione con la ricerca della filosofia nazionale croata.

Le parole chiavi: Alberto Bazala; storia della filosofia; compito; approccio positivo; filosofia nazionale croata 


\section{NoTA INTRODUTTIVA}

Sebbene Alberto Bazala ${ }^{1}$, come ha osservato bene il filosofo croato Vladimir Filipovic (1906-1984), non scrisse un gran numero di libri che potevano essere stampati come opere sistematiche in diversi volumi (opera omnia), non era nemmeno un teorico speculativo né uno studioso di gabinetto a chi biblioteche e banchi sostituirebbero l'attività nella vita culturale ${ }^{2}$ e politica, tuttavia, si deve dire che la sua Storia della filosofia in tre volumi - fino ad oggi è un'opera unica nella storia della filosofia croata e oltre - rappresenta, da un lato, il lavoro della sua vita e, dall'altro, lo affianca ai più grandi filosofi e ricercatori croati della storia della filosofia nel $20^{\circ}$ secolo, e non solo nella Croazia ma anche oltre.

Ispirato alle idee del suo insegnante Franjo Markovic (18451914), Bazala per l'educazione filosofica scrisse una storia della filosofia in tre volumi. Per la prima volta nella nostra regione ${ }^{3}$, ha studiato da solo il vasto materiale di duemila e mezzo anni di sviluppo del pensiero filosofico e ha trovato termini adeguati

Alberto Bazala è uno dei filosofi croati la cui vita e lavoro sono stati in qualche modo esplorati. Numerosi articoli scientifici sono stati scritti su di lui, così come alcuni aspetti della sua filosofia, ma il giudizio di Ljerke Schiffler è ancora valido: "Tra le figure significative della nostra storia e filosofia culturale è Alberto Bazala, un pensatore che non ha ancora una rappresentazione sintetica, e anche il suo lavoro e la sua opera scritta non hanno valutazioni globali". Ljerka Schiffler, Filozofijsko nastojanje Alberta Bazale, in: Prilozi za istraživanje hrvatske filozofske baštine, vol. 14 (1988) n. 27-28, p. 101. Ecco alcuni lavori su Bazala nella lingua croata: Zlatko Posavac, Estetički nazori Alberta Bazale u doba hrvatske Moderne, in: Prilozi za istraživanje hrvatske filozofske baštine, vol. 14 (1988) n. 27-28, pp. 135-158.; Boris Kalin, Bazala i nastava filozofije, in: Prilozi za istraživanje hrvatske filozofske baštine, vol. 14 (1988) n. 27-28, pp. 165-174.; Franjo Zenko, Bazala o problemu ličnosti/osobnosti, in: Prilozi za istraživanje hrvatske filozofske baštine, vol. 23 (1997) n. 45-46, pp. 119-128 ecc.

2 Cf. Vladimir Filipović, Filozofska misao Alberta Bazale. Povodom stote obljetnice rođenja (1877-1947), in: Prilozi za istraživanje hrvatske filozofske baštine, vol. 4 (1978) n. 7-8, p. 7.

3 E necessario menzionare qui il filosofo ecclesiastico e sacerdote Francesco Šanc che pubblicò due volumi sulla storia della filosofia, mentre il terzo che prevedeva a causa della morte non finì. Usp. Francesco Šanc, Povijest filozofije. I. dio: Filozofija starih Grka i Rimljana, Knjižnica života, Zagabria, 1942; Francesco Šanc, Poviest filozofije II. dio. Od renesanse do Kanta, Knjižnica života, Zagabria, 1943. Abbiamo presentato entrambi questi lavori sulla storia della filosofia, oltre alla vita di Šanc in nostro lavoro scritto: Ivan Macut, Filozofija u Nezavisnoj Državi Hrvatskoj [Filosofia nello Indipendente Stato Croato], Školska knjiga, Zagabria, 2018, pp. 251-262. Altre opere nel campo della storia della filosofia nella lingua croata includono il seguente autore: Branko Bošnjak, Povijest filozofije (I - III, 1990 - 1993). Lui ha presentato anche tutta la storia della filosofia in tre volumi, ma il suo approccio è diverso da quello di Bazala e Šanc. 
nella lingua croata che gli ha permesso di creare molte innovazioni linguistiche. Lui è riuscito alla fine a tradurre diversi concetti e pensieri filosofici nella lingua croata $^{4}$. Pertanto, Vladimir Filipovic può giustamente concludere e noi concordiamo pienamente con questa conclusione, quando scrive: "Se non avesse fatto nient'altro nella vita ma soltanto avesse arricchito il nostro linguaggio con questa creazione, il suo nome sarebbe stato profondamente inscritto nello sviluppo delle nostre possibilità filosofiche espressive" 5 .

Alla fine, l'importanza di quest'opera di Bazala è evidente anche nel fatto che, in un certo senso, si trova all'inizio del suo insegnamento e della sua carriera filosofica, e non alla fine quando potremmo aspettarci una opera di questa portata e importanza.

\section{In breve sulla vita e l'opera di Alberto Bazala}

Alberto Bazala è nato il 13 luglio 1877 a $\mathrm{Brno}^{6}$. Si è laureato in Filosofia e Filologia classica a Zagabria e ha conseguito il dottorato il 1900. Nel 1904 divenne un docente privato con il lavoro di abilitazione intitolato Il lavoro morale e filosofico di Marulić. Ha continuato i suoi studi di filosofia in Germania alla scuola dei "famosi professori tedeschi". Divenne professore ordinario nel 1912, quando fu pubblicato il terzo volume della sua opera più famosa, La storia della filosofia. Nello spirito dell'idea dell'Illuminismo, ha fondato e diretto l'Università del popolo ed è stato presidente della Matica hrvatska. Come presidente di

4 Sulla nostra terminologia filosofica croata e sullo straordinario contributo di Bazala in questo contesto, traiamo una breve conclusione dalla nostra filosofa Maria Brida: "La terminologia di Bazala, implementata in modo inequivocabile e coerente in tutte le epoche filosofiche, fino agli anni '20 del nostro secolo, quindi, ha dato un contributo significativo allo sviluppo del nostro pensiero filosofico. In alcuni casi ha anche reso visibile l'eccezionale capacità della lingua croata o serba di esprimere concetti filosofici". Marija Brida, Naša filosofijska terminologija na prijelomu stoljeća i Bazalin udio, in: Prilozi za istraživanje hrvatske filozofske baštine, vol. 14 (1988) n. 27-28, p. 163.

5 V. Filipović, Filozofska misao Alberta Bazale, p. 13.

6 Qui seguiamo la sua vitta secondo il nostro libro: Ivan Macut, Hrvatska filozofija od obnove Zagrebačkog sveučilišta 1874. do osnutka Nezavisne Države Hrvatske 1941., Split, 2018, pp. 102-106., recuperiamo anche i dati in parallelo: http:// www.enciklopedija.hr/natuknica.aspx?id=6396 (accesso 1. 11. 2019) Di Bazala e la sua filosofia in un periodo storico specifico (1941 - 1945) abbiamo scritto nel nostro libro: I. Macut, Filozofija u Nezavisnoj Državi Hrvatskoj [Filosofia nello Indipendente Stato Croato], pp. 179-190. 
L'Accademia jugoslava delle Scienze e delle Arti (JAZU), è noto per i suoi discorsi Sulla libertà della scienza e dell'arte (1934) e Il significato dell'arte nella vita della nazione (1935). Muore il 12 agosto 1947 a Zagabria.

Le sue opere più importanti sono: La storia della filosofia in tre volumi, La radice metallogica della filosofia (1924); Sull'idea della filosofia nazionale; Studi filosofici, 2. Coscienza e mondo, soggetto e oggetto.

2. Brevemente sull'opera la storia della filosofia. Volume I. LA STORIA DELLA FILOSOFIA POPOLARE DEI GRECI

Bazala scrisse la Storia della filosofia $(1906,1909,1912)$ prima delle sue principali opere teoriche. Quindi questa opera non contiene le sue opinioni originali o i suoi giudizi filosofici sulla storia della filosofia. Nello scrivere la sua opera, Bazala aveva a sua disposizione tutte le opere di base della letteratura storica e filosofica. Inoltre, la Storia della filosofia mostra che si tratta di un'opera altamente appresa in cui l'erudizione e l'apprendimento dell'autore sono venuti alla luce in modo speciale. Ha studiato una vasta gamma di materiale di ricerca storica e filosofica e tutta la letteratura pertinente è stata citata o detta esplicitamente ${ }^{7}$.

Sebbene questa, come abbiamo già detto, sia la prima storia della filosofia in lingua croata, comunque: "è necessario sottolineare che non si potrebbe pensare che questo lavoro (...) sia ormai obsoleto o, per di più, solo un degno lavoro per un iniziatore nella materia. In effetti, nonostante queste circostanze, che

7 Cf. Goran Gretić, O ulozi i značenju povijesti filozofije [Sul ruolo e il significato della storia della filosofia], in: Prilozi za istraživanje hrvatske filozofske baštine, vol. 14 (1988) n. 1-2, pp. 127-130. "Per il nostro patrimonio culturale, questo lavoro è di grande importanza, non solo come un prezioso documento della nostra tradizione filosofica, ma come oggi un lavoro scientifico significativo. Molti capitoli della Storia della filosofia di Bazala rappresentano le pagine più belle della nostra letteratura filosofica, ad esempio i capitoli su Platone, Stoi, Kant e Fichte. Per noi oggi, il suo linguaggio originale e peculiare, che è lontano da qualsiasi tipo di gergo, può essere particolarmente incoraggiante per noi, e che oggi grava anche sulla nostra scrittura. (...) Con la sua Storia della filosofia, ha dato al pubblico culturale un'introduzione molto professionale e affidabile allo studio della filosofia, e allo stesso tempo ha fissato un alto livello di lavoro storico e filosofico. In particolare, il suo lavoro e i suoi quarant'anni di lavoro come professore di filosofia hanno creato le condizioni e permesso lo sviluppo in mezzo a noi di un'atmosfera filosofica, da Franjo Markovic a lui, che pensiero filosofico era interamente a livello europeo." G. Gretić, O ulozi $i$ značenju povijesti filozofije [Sul ruolo e il significato della storia della filosofia], pp. 133-124. 
davvero non dovrebbero essere sottovalutate, è un'opera, con il lavoro di un solo autore, interamente al livello dei risultati della ricerca filosofica storica europea dell'epoca. A parte questo, va detto che non c'erano molte opere così ambiziose e complete sia prima che oggi."

Poiché questo lavoro riguarda solo il primo volume della Storia della filosofia, lo presenteremo brevemente prima di entrare nella parte centrale del nostro lavoro.

Il titolo completo del libro recita: La storia della filosofia. Volume I. Una storia della filosofia popolare dei greci. L'opera è stata stampata nel 1906 a Zagabria9 ${ }^{9}$ Come suggerisce il titolo, il libro è interamente dedicato alla storia della filosofia popolare greca. Comprende quattro sezioni: La prima sezione contiene un discorso sullo sviluppo della filosofia greca e delle opportunità educative in Grecia prima della continuazione della filosofia (visione omerica della vita, i Saggi e Pitagora). La seconda sezione è dedicata al discorso del periodo cosmologico (filosofi ionici, Eraclito ed Eleusiti; Mediatori, elementi, atomi e numero - Empedocle, Anaxagoras, Leukipus e Democritus e Pitagorici). La terza sezione tratta del periodo antropologico (Lo sviluppo delle opportunità educative nel V secolo - Sofisti, Socrate e Socratici). La quarta sezione parla dell'età del sistema, Platone (la vecchia accademia) ed Aristotele (Peripatetica) sono stati discussi e presentati qui.

Il punto di partenza per Bazala è, basandosi sulla ricerca e sull'opinione di Zeller, che la filosofia popolare greca si autoimposta: "I Greci non trapiantarono alcun sistema dall'est, ma piuttosto adattarono il ricevuto dall'Oriente alla loro natura, creando così costruire una filosofia popolare greca" ${ }^{10}$. Inoltre, molte somiglianze che esistono in aree educative separate e sebbene $\mathrm{i}$ pensieri di base possano essere ripetuti, secondo Bazala e non devono essere dedotti dalla connessione storica perché potrebbero essere emersi da soli. ${ }^{11}$

Ibid., p. 130.

Alberto Bazala, Povjest filozofije. Svezak I. Povjest narodne filozofije grčke, [La storia della filosofia. Volume I. La storia della filosofia popolare dei greci], Dionička tiskara u Zagrebu, Zagabria, 1906.

$10 \quad$ Ibid., p. 57.

11 Cf. Ibid., p. 57. "La relazione tra la storia della filosofia e il pensiero filosofico è, come è noto, specifica rispetto alla filosofia stessa, e in particolare rispetto ad altre scienze. Mentre nelle varie scienze, in generale, la loro storia è più o meno la storia delle opinioni precedenti e delle intuizioni prevalenti o confutate, in filosofia la situazione è piuttosto l'opposta. Vale a dire, la storia della filosofia è una 
Prima di intraprendere uno studio di esaminare quale è il compito della storia della filosofia stessa, va notato, per motivi di interesse, che alla fine del libro, l'autore ha scritto una nota in vista dei numerosi errori tipografici nel libro. La ragione perché sono stati molti errori nel libro è che Bazala in questo tempo non era a Zagabria e così non poteva correggere gli errori.

\section{IL COMPITO DELLA STORIA DELLA FILOSOFIA}

Introduzione al primo volume dell'opera filosofica più importante della sua vita La storia della filosofia è stata divisa in tre parti: 1. Che cos'è la filosofia? 2. Il compito della storia della filosofia e 3. La filosofia nelle nazioni orientali. Poiché il tema centrale di questo lavoro è il compito della storia della filosofia come lo comprende Bazala, non discuteremo in modo specifico delle altre due parti dell'introduzione di Bazala in questo nostro lavoro.

\subsection{L'importanza della storia della filosofia}

Per quanto riguarda il compito della storia della filosofia, Bazala è convinto che per nessuna scienza sia così importante conoscere la sua storia come lo è per la filosofia. Un matematico può essere un buon matematico senza alcuna conoscenza della storia della matematica, e così è con altre scienze come: la fisica, la geografia, ecc., poiché la storia stessa non ne costituisce una parte essenziale. Mentre quando si parla di storia della filosofia, allora, Bazala crede, le cose stanno diversamente. Vale a dire, la storia della filosofia fa parte della filosofia, e dal punto di vista storico è lo sviluppo della mente umana, e alcuni dei momenti di questo sviluppo sono sistemi filosofici ${ }^{12}$.

parte indistinguibile e costitutiva del pensiero filosofico in quanto tale, e da ciò deriva a prima vista il fatto che la filosofia conosce nel corso della sua lunga storia un numero davvero piccolo di questioni completamente nuove. (...) Ecco perché la storia della filosofia è uno sforzo e un segnale per comprendere noi stessi e il nostro destino nel mondo, uno sforzo che non può mai essere obsoleto o completamente superato, ma solo ripensato, riprovato". G. Grgetić, O ulozi i značenju povijesti filozofije [Sul ruolo e il significato della storia della filosofia], p. 127.

12 Cf. Ibid., pp. 19-20. 


\subsection{Il doppio compito della storia della filosofia}

Bazala è convinto che la storia della filosofia influenza lo sviluppo della filosofia stessa, cioè senza la storia della filosofia non esiste filosofia. Questo sviluppo è dimostrato in due direzioni: "Nel positivo come l'accertamento della verità, nel negativo come prevenzione dell'illusione". La filosofia alla base di Bazala mira a racchiudere l'intera realtà con un solo sguardo e creare un'immagine del mondo ${ }^{13}$. Queste immagini filosofiche del mondo hanno una certa permanenza, come gli artisti classici, e non invecchiano rapidamente come le teorie naturali. Questi dipinti hanno sempre un valore definito, ma anche nello stesso tempo relativo: "In nessun luogo esistono idee sbagliate così istruttive, da nessuna parte è solo nuovo per completare e perfezionare il vecchio - anche se sembra del tutto nuovo almeno abbattere tutto ciò che è esistito prima - proprio come in filosofia, nella storia della filosofia. I sistemi filosoficamente si rovesciano a vicenda, ma non si distruggono a vicenda: una visione del mondo, rovesciando l'altra, sceglie ciò che gli sembra migliore e si basa quindi su di esso". ${ }^{14}$

Secondo il nostro filosofo, la storia della filosofia ha un duplice compito: poiché è la realtà un grande enigma che l'umanità cerca di svelare e penetrare a poco a poco, la storia della filosofia deve prima spiegare l'immagine individuale come è concepita dallo scrittore e mostrare la sua esistenza e connessione con il passato ${ }^{15}$, e dopo, nella seconda fase, determinare il valore dell'intero sistema o del pensiero individuale in esso per lo sviluppo dell'aspirazione generale dell'umanità"16.

A volte i filosofi esprimono il meglio di se stessi e lo storico volge lo sguardo proprio su questi rari pensieri. È interessante

13 Bazala nel lavoro sotto il titolo O ideji nacionalne filozofije [Sull'idea della filosofia nazionale] scrive: “(...) la storia della filosofia come scienza, che non fornisce in alcun modo un resoconto unico della figura spirituale di alcun individuo di significato filosofico, di periodo o di tipo nazionale, ma enfatizza, soprattutto, solo per mezzo del quale estrarre il profilo, ma non dà l'intero aspetto vivente del loro". Alberto Bazala, O ideji nacionalne filozofije [Sull'idea della filosofia nazionale], in: Alma mater croatica, 1937/1938, n. 8-10, p. 225.

14 A. Bazala, Povjest filozofije. Svezak I. [La storia della filosofia. Volume I.], p. 21.

15 "Affinché la storia della filosofia sia in grado di soddisfare il suo primo compito, è necessario assumere il punto di vista di un singolo filosofo, immergersi il più profondamente possibile nella sua dottrina e spiegare i suoi pensieri e mostrare quali conseguenze li seguono e a quale scopo mirano". Ibid, pp. 21-22.

16 Cf. Ibid., p. 21. 
che Bazala stia cercando il meglio nei sistemi filosofici, e quello che è il miglior dovrebbe essere il criterio in base al quale il sistema stesso può essere valutato: "Lui [lo storico, op.a.] giudicherà i sistemi meglio se visti dai loro grandi lati, piuttosto che dai loro piccoli" e continua dicendo che, rispetto ai pensatori filosofici, lo storico: "Deve cercare nelle loro opere le manifestazioni più straordinarie dei propri pensieri. In filosofia come nell'arte, molte critiche non riguardano i difetti ma la bellezza". ${ }^{17}$

\subsection{Fattore socio-culturale}

Bazala sottolinea che la filosofia non è un insieme di pensieri spiritosi (placita philosophorum) e delusioni dell'umano, ma è sempre determinata dalle aspirazioni dei bisogni della propria età, e dipende da loro quali domande verranno in superficie e decidono su quali domande sollevare e nella quale direzione andranno. Pertanto, le visioni filosofiche di un'età non sono solo il lavoro dell'individuo: "Il filosofo si alza spesso molto al di sopra di ciò che lo circonda, distinguendosi con uno sguardo più profondo, un sentimento più nobile e una certa volontà. Riconoscere questa parte del lavoro dell'individuo nel creare una visione della vita, non significa ancora separarlo da tutto ciò che lo circonda e dalle opportunità delle aspirazioni e dei bisogni generali contemporanei". ${ }^{18}$

Bazala pensa che nessun grande lavoro venga svolto senza i suoi predecessori che preparano la strada e il sistema deve essere considerato come una perfezione del suo tempo. La storia della filosofia secondo Bazala deve tenere conto dei seguenti fattori: il sistema filosofico individuale è il lavoro di molti fattori, e con essi tutta la storia della filosofia deve contare; rimuovere l'estremo dell'individualismo completo secondo il quale la storia della filosofia sarebbe semplicemente una storia dei pensieri e delle frasi argute delle singole persone, in nessun ordine, dipendente solo dal mero tempo, dalla possibilità o dal capriccio. Bazala conclude che un sistema individuale è in realtà uno sviluppo della coscienza generale, un'immagine del mondo e della vita che dipende da insegnamenti precedenti, dal significato e dalla natura delle persone stesse. ${ }^{19}$

\footnotetext{
17 Ibid., pp. 22-32.

18 Ibid., p. 23.

19 Cf. Ibid., p. 23.
} 


\subsection{Tripla interpretazione dei sistemi filosofici}

Secondo Bazala, devono essere presi in considerazione i seguenti tre fattori: a) interpretazione psicologica: la storia della filosofia descrive l'emergere di un particolare sistema, e la personalità del filosofo e le sue relazioni ed esperienze personali dovrebbero essere enfatizzate perché sono le cause e le ragioni più vicine alla sua opinione; b) interpretazione storico-culturale: qui verranno evidenziate le circostanze di un particolare momento, posizione geografica, ordine politico, stato economico, altezza della cultura mentale e sviluppo di alcune scienze; c) interpretazione pragmatica: qui l'individuo guarderà indietro e sul passato costruirà suo pensiero. Questioni irrisolte come l'eredità vanno dalla generazione in generazione a cui queste nuove generazioni continuano a pensarci, ed è per questo che alcuni sistemi filosofici non formano entità chiaramente separate, ma sono piuttosto una serie continua ${ }^{20}$. Tenendo presente tutto ciò, Bazala conclude: "Quindi la storia della filosofia non è un insieme di opinioni private o pensieri strani e casuali, ma lo sviluppo del pensiero dell'illuminazione umana, a cui gli individui hanno a volte dato una forma tipica definita e chiara", e scrive ancora un po' più avanti: "I sistemi filosofici, come menzionato sopra, non sono solo un riflesso del pensiero generale, ma enfatizzano i pensieri individuali, le aspirazioni e i bisogni degli individui, che conferiscono all'intero sistema una caratteristica individuale. Questo fattore individuale nello sviluppo dello spirito umano non deve trascurare la storia della filosofia, se vuole adempiere pienamente al suo primo compito". ${ }^{21}$

\subsection{Critica del singolo sistema}

Un altro compito che appartiene alla storia della filosofia è: la critica di un sistema particolare. Il criterio principale per la valutazione è la questione di quanto un determinato sistema abbia contribuito allo sviluppo del pensiero filosofico. Questa critica va secondo Bazala in due direzioni: in negativo - la critica deve evidenziare le carenze e le idee sbagliate del singolo sistema, in positivo - deve trovare vantaggi e influenza. Per quanto riguarda

\footnotetext{
$20 \quad$ Cf. Ibid., p. 24.

21 Ibid., pp. 24-25.
} 
i principi in base ai quali verrà giudicato un sistema, Bazala trova che sono difficili per determinare sicuramente. "E così costante che le critiche devono essere scientifiche, e quindi generali, e quindi non dovrebbero essere supportate dogmaticamente da un certo punto di vista, ad es. una visione che considera in anticipo alcune delle affermazioni come vere; inoltre, questo giudizio non deve essere la base di una particolare convinzione filosofica dello storico, né dovrebbe in definitiva essere usato filosoficamente da un particolare sistema. Di conseguenza, a un giudizio scientifico resta solo una visione che si appoggia sul sistema stesso, una visione di critica immanente, che è chiamata così, perché rimane all'interno del sistema (immaneo)". ${ }^{22}$

Per quanto riguarda la direzione negativa della critica e del giudizio di determinati sistemi, Bazala ritiene che la storia della filosofia abbia il compito in un particolare sistema di scoprire le delusioni, in un duplice modo: se un'affermazione è in sé sbagliata, allora tale illusione è chiamata un errore di principio; un altro tipo di illusione nasce dall'applicazione errata di principi e incoerenze di pensiero e ragionamento, e la storia della filosofia deve determinare in che misura i pensieri di dei singoli filosofi concordano tra loro e quanto si sono riusciti a mettere in un insieme armonico e non contraddittorio. Il principio di tale giudizio è: coerenza logica formale (consequenzialità). ${ }^{23}$

Quando vengono soddisfatte queste due attività, devono essere prese in considerazione le basi del sistema in questione. Nello specifico, una o più affermazioni vengono utilizzate come base per ciascun sistema, su cui si basa effettivamente l'intero pensiero, e queste basi dovrebbero essere illuminate. Bazala è convinto che anche nei fondamenti che sono sbagliati, c'è sempre qualche valore e verità in essi, perché l'assurdo non può essere né compreso né pronunciato e secondo Bazala un sistema basato su tali affermazioni non può né prendere vita né svilupparsi affatto. "Le idee sbagliate di principio consistono in una verità incompleta, ma non in invalidità assoluta." ${ }^{24}$

Il compito positivo di valutare un particolare sistema di pensiero è determinare il sistema in questione secondo il principio della fertilità intellettuale, vale a dire quanto hanno influenzato lo

\footnotetext{
22 Ibid., pp. 25-26.

23 Cf. Ibid., p. 26.

24 Ibid., p. 26.
} 
sviluppo e il perfezionamento delle visioni del mondo e della vita. Sotto questo aspetto le idee sbagliate sono importanti, poiché sono state spesso ridotte alla giusta misura a nuovi pensieri e nuove opinioni, e Bazala considera l'affermazione di Windelband, secondo cui grandi idee sbagliate sono più importanti nella storia della filosofia che di piccole verità, come giusta. ${ }^{25}$

\subsection{Il valore della storia della filosofia}

La storia della filosofia, secondo Bazala, ha valore, innanzitutto, rispetto alla filosofia stessa, poiché la filosofia non può essere studiata senza conoscerne la storia. Inoltre, la storia della filosofia ci mette in relazione con grandi pensatori. Ci ispira con l'ammirazione e gratitudine verso i nostri antenati e nello stesso tempo dimostra che i filosofi sono amici l'uno con l'altro nell'esplorazione dei problemi fondamentali del mondo e dell'uomo. In questo contesto, la storia della filosofia aiuta anche nella ricerca della verità. ${ }^{26}$

\subsection{Problemi filosofici}

Bazala ritiene che la storia della filosofia affronta due problemi: il problema teorico e pratico. Per quanto riguardano i problemi teorici, quindi, il problema più significativo è la cognizione, esamina la stessa cognizione, la sua esistenza e la sua sicurezza, ed anche i confini. E questo problema cognitivo che sta alla base di tutti gli altri problemi filosofici e, soprattutto, della metafisica che mira a conoscere tutto ciò che esiste. Tra i problemi pratici, il posto più importante secondo Bazala è occupato da problemi etici (sulla vita reciproca delle persone e la loro azione secondo determinati principi). Questi problemi sono collegati con la sociologia (sulla vita e al lavoro della società), legge (filosofia giuridica), Arte (estetica, filosofia dell'arte) e religiosità (filosofia della religione). ${ }^{27}$

Bazala la filosofia della volontà ritiene più importante della filosofia della mente ${ }^{28}$, in questo spiega con seguente parole:

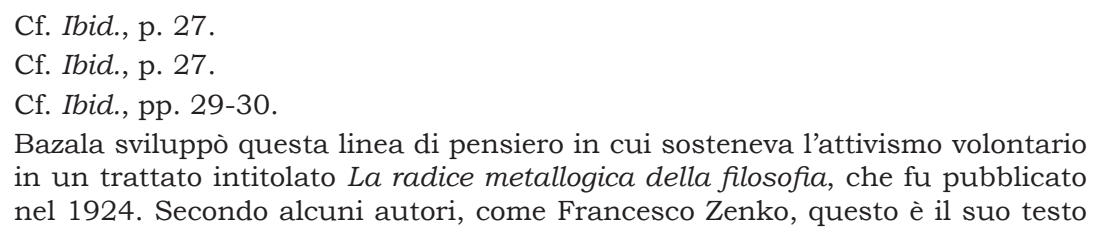
in un trattato intitolato La radice metallogica della filosofia, che fu pubblicato nel 1924. Secondo alcuni autori, come Francesco Zenko, questo è il suo testo 
"Quindi, sul problema cognitivo, riposa il problema metafisico, come la filosofia della mente, e di nuovo sulla metafisica riposa il problema etico come la filosofia della volontà. Questo non vuol dire, naturalmente, che l'etica, l'estetica, la filosofia giuridica e la filosofia della religione come scienze hanno la loro origine nella metafisica stessa, (...) ma solo che molto, quasi interamente, dipenderà dalla soluzione di problemi teorici le soluzioni dai problemi pratici - che poi, nel loro insieme, danno forma e contenuto alla vita e all'azione, e quindi creano una visione della vita. Sotto questo aspetto, la visione del mondo è la base della visione della vita, la filosofia della mente è la base della filosofia della volontà, che è più che la filosofia della mente". ${ }^{29}$

\subsection{Una divisione della storia della filosofia}

L'ultimo titolo di questo capitolo discute la divisione della storia della filosofia. Bazala comincia dal fatto che la filosofia si cambia di volta in volta, vale a dire le veniva sempre assegnato un compito diverso e il suo contenuto era diverso, e questo fatto di cambiamento della filosofia era anche influenzato dalle opportunità nella società, dallo stato, dai progressi nelle scienze, dalla forza del sentimento religioso, ecc. Questi sono i motivi per cui esistono periodi nella storia della filosofia, che generalmente coincidono con sezioni più grandi e più importanti della storia del mondo. Infine, Bazala accetta anche la divisione generale della storia della filosofia in: la storia della vecchia, media e nuova era. ${ }^{30}$

Conclusione: L'APPROCCIO POSITIVO Di BAZALA ALLA STORIA DELLA FILOSOFIA

Bazala, come abbiamo sottolineato in precedenza, è uno dei pochissimi ricercatori nel nostro paese croato, e anche il primo tra loro, che con il suo lavoro ha sistematicamente coperto l'intera storia della filosofia. Il fatto che Bazala sia stato intellettualmente capace di fare questo è dimostrato dal suo studio della

più singolare e filosoficamente rilevante. Cf. Francesco Zenko, Alberto Bazala, in: Francesco Zenko (ed.), Novija hrvatska filozofija [La filosofia croata recente], Zagabria, 1995, p. 201. E in questa crestomazia filosofica che il testo filosofico di Bazala viene pubblicato nella sua totalità.

29 A. Bazala, Povjest filozofije. Svezak I. [La storia della filosofia. Volume I.] p. 30.

$30 \quad$ Cf. Ibid., p. 30. 
filosofia che in Croazia sia all'estero, come dai suoi numerosi lavori di ricerca in cui discute questioni particolari della storia filosofica mondiale e nazionale. Riteniamo che il suo lavoro in tre volumi sotto il titolo Storia della filosofia possa essere giustamente definito il suo principale lavoro storico-filosofico, senza ridurre l'importanza delle altre sue opere filosofiche.

Nel complesso, possiamo giustamente sottolineare che Bazala, da un lato, sottolinea un posto insostituibile per la storia della filosofia ${ }^{31}$ per quanto riguarda la filosofia in quanto tale, cioè, quando afferma che senza la storia della filosofia non esiste filosofia e che senza la conoscenza della storia della filosofia, la filosofia non può essere studiata e, dall'altro, sottolinea che lo storico della filosofia dovrebbe avere un approccio positivo piuttosto che negativo alla realtà filosofica e al giudizio dei sistemi filosofici. Mentre non è fondamentale per un matematico conoscere la storia della matematica per essere un matematico eccellente, quando si tratta di filosofia, non è così, pensa Bazala.

Lo storico della filosofia Bazala sottolinea che la storia della filosofia non distrugge, ma piuttosto modella l'individuo e il suo pensiero. Invece il pensiero del singolo filosofo scompare nella diversità del pensiero filosofico, emerge chiaramente e si mostra nella sua chiarezza tra gli altri pensieri e come tale può essere confrontato con gli altri. Bazala pensa che la storia della filosofia, per quanto riguarda l'individuo con il suo pensiero-filosofia, mostra anche la sua originalità e novità nella storia del pensiero filosofico. Non pone così tanta enfasi su Bazala sugli errori dei singoli filosofi, quanto sul fatto che anche questo pensiero a volte fuorviato può essere fruttuoso. In questo contesto questo pensiero umano sbagliato possa portare a uno sviluppo nuovo e più forte. Un pensiero fuorviato può anche essere un potente driver per coloro che lo incontrano per un nuovo pensiero, e quindi questo pensiero fuorviato è anche responsabile, in un certo senso, dell'emergere di un nuovo, vero pensiero.

Bazala è consapevole che il pensiero filosofico umano è modellato dalle particolari circostanze storiche in cui ogni individuo o società vive e opera. Pertanto, la storia non può essere ignorata mentre si esplora la storia del pensiero umano.

31 In un altro posto Bazala scrive: "La storia del pensiero umano è una serie di tentativi di fuga dal labirinto del mondo e della vita". Alberto Bazala, Svijet $i$ život u perspektivi fizikalnog racionalizma. 300. godišnjica rođenja Baruha Spinoze, in: RAD JAZU, libro 245 (1933), p. 201. 
Individuo pensante - il filosofo - non modella il suo pensiero indipendentemente dalle circostanze sociali, non lo modella indipendentemente da altre personalità forti, in particolare i filosofi. Ecco perché in ogni epoca storica è cruciale trovare e comprendere il pensiero dei grandi filosofi, ma anche metterlo in interrelazione, con pensatori più piccoli. Comune sia ai "piccoli" che ai "grandi" filosofi è la ricerca della verità. La verità viene lentamente rivelata attraverso l'individuo alla comunità, ma anche viceversa, e la storia della filosofia nel contesto della ricerca della verità è un grande aiuto per tutti. ${ }^{32}$

Alla fine, vogliamo solo indicare brevemente la riflessione di Bazala nel contesto della filosofia nazionale e della sua storia particolare. Quando si tratta della storia della filosofia, ovviamente, non si possano ignorare i suoi discorsi e scritto sulla storia della filosofia croata. Da un lato, Stefano Zimmermann ha insistito su particolari accenti e aspetti del cristianesimo dell'Europa occidentale nella scrittura e nella ricerca della storia nazionale, dall'altro Alberto Bazala la sua concezione basa su un concetto secolare $^{33}$. Bazala sostenne inoltre che la storia nazionale della filosofia è importante anche per lo spirito nazionale o per il pensiero nazionale. Bazala, e conosciuto, non aveva scritto una storia della filosofia nazionale. In questo contesto, il nostro rinomato ricercatore dell'eredità filosofica croata Zlatko Posavac afferma anche che Bazala non solo non ha affrontato a fondo lo studio della filosofia nazionale ${ }^{34}$ e quindi la sua sistematizzazione, ma la sua preoccupazione per la filosofia nazionale si è trasformata nella filosofia del nazionale. ${ }^{35}$

32 "Lo sforzo filosofico di Bazala assume la dignità della storia, indipendentemente dal risultato stesso. $\mathrm{E}$ in questa avventura spirituale dell'essere culturale come storico (il presente è indistruttibile nel passato, il passato contenuto nel presente), attraverso questa forza vitale, è resa possibile tutta la cognizione amata". Lj. Schiffler, Filozofijsko nastojanje Alberta Bazale, p. 106.

33 Cf. Zlatko Posavac, Kruno Krstić kao istraživač i povjesnik hrvatske filozofije, in: Prilozi za istraživanje hrvatske filozofske baštine, n. 43-44 (1996), p. 268.

34 Sulla idea della filosofia nazionale di Bazala raccomandiamo un lavoro eccellente: Franjo Zenko, O ideji (hrvatske) nacionalne filozofije u Alberta Bazale, in: Prilozi za istraživanje hrvatske filozofske baštine, vol. 14 (1988), n. 1-2, pp. 109-126.

35 Cf. Ibid., p. 288. Evidenziamo anche il suo discorso alla solenne sessione di JAZU del 4 febbraio 1935, intitolata A proposito del pensiero jugoslavo. G. Gretic considera questo lavoro come segue: "Questo trattato di Bazala sul pensiero jugoslavo è ovviamente una sorta di concretizzazione della sua idea di filosofia nazionale, ma mostra anche e chiarisce che l'idea di filosofia nazionale è in realtà l'idea di filosofia del popolo, di un certo ambiente spirituale-culturale, basata su 


\section{THE TASK OF THE HISTORY OF PHILOSOPHY ACCORDING TO ALBERT BAZALA IN THE FIRST VOLUME OF HIS BOOK HISTORY OF PHILOSOPHY}

\section{Summary}

In this paper we are talking about the task of the history of philosophy according to the Croatian philosopher Albert Bazala, which is written about in his important work History of Philosophy. After first introductory remarks and a brief account of the life and work of Albert Bazala in the first chapter, we move on to the second chapter in which we schematically present Bazala's work entitled History of Philosophy, Volume I, History of the Folk Philosophy of Greece. The third, and also the main chapter deals with the task of the history of philosophy, as Bazala sees it, and is divided into the following topics: the importance of the history of philosophy; the double task of the history of philosophy; sociocultural factor; triple interpretation of philosophical systems; criticism of a particular system; the importance of the history of philosophy; philosophical problems, and finally, the division of the history of philosophy. In the conclusion of the paper, we systematically present and highlight the main points of Basala's positive approach to the history of philosophy, and briefly outline his relation to the research of national Croatian philosophy.

Keywords: Albert Bazala; history of philosophy; task; positive approach; national philosophy

una serie di determinanti sia storici che culturali." Goran Gretić, Albert Bazala - utemeljenje i konstitucija "Ideje nacionalne filozofije", in: Prilozi za istraživanje hrvatske filozofske baštine, vol. 16 (1990), n. 31-32, p. 59. A causa di questi atteggiamenti jugoslavi, Bazala ha entrato in conflitto con il suo contemporaneo e questo fu sacerdote e filosofo Stefano Zimmermann. 